\section{Can the weather influence arthritis symptoms?}

\author{
Wai Chin Li, Ye Zhang \\ Centre for Education in Environmental \\ Sustainability, Department of Science \\ and Environmental Studies, The Hong \\ Kong Institute of Education, Hong Kong
}

\begin{abstract}
By reviewing twelve previous studies on the relationship between the weather and arthritis symptoms, this article summarized the major findings on this controversial topic. It has been supported by most of the studies that arthritis symptoms are associated with weather conditions. Factors such as barometric pressure and temperature were generally believed to be related to arthritis symptoms. However, controversies arose at minor levels. Whether the weather-symptom relationship depends on the specific type of arthritis, weather variables, demographic and pathological heterogeneity of the patients is still a matter of debate. This article calls for standardization in future research design, and hope the mechanism underlying the weather-symptom association will be found.
\end{abstract}

\section{Introduction}

Arthritis is one of the most common chronic diseases we can find in daily life. From a clinical point of view, pain is the major complaint by individuals who have arthritis, but, interestingly, many patients frequently perceive a relationship between weather conditions and arthritic symptoms. According to a telephone questionnaire done in Australia, $92 \%$ of the participants thought their symptoms would be affected by the weather change. Moreover, $48 \%$ of the participants believed that the weather can be predicted by their feeling of symptoms. ${ }^{1}$

Arthritis is a form of joint disorder which involves inflammation of one or more joints. Joints are complex structures consisting of bone, cartilage, ligaments, capsule and synovium. Problems in different parts of a joint will cause different types of arthritis. It is estimated that there are more than 100 types of arthritis, but the most common forms are osteoarthritis (OA) and rheumatoid arthritis (RA). OA is a complex disease of the entire joint, affecting bone, cartilage and synovium. ${ }^{2} \mathrm{~A}$ hallmark of the disease is progressive degeneration of articular cartilage and subsequent joint space narrowing. In addition to genetic risk factors, known risk factors for $\mathrm{OA}$ are age, significant trauma, altered gait, altered bio- mechanics, and excessive loading on the affected joint. ${ }^{3}$ RA is a chronic immune-mediated disease primarily affecting the synovial membrane of diarthrodial joints, although other compartments may be involved in course of the disease. ${ }^{4,5}$ The synovial lesion in RA is characterized by resident cell proliferation, increased vascularization and infiltration of innate and adaptive immune cells. ${ }^{6,7}$ Collectively, localization of inflammation to the synovium is primarily responsible for the deregulated cellular and molecular mechanisms that ultimately lead to the typical signs and symptoms of RA. ${ }^{8}$ According to the report of Arthritis Foundation, ${ }^{9}$ there are about 27 million Americans affected by $\mathrm{OA}$ while another 1.5 million affected by RA. Patients of both types have symptoms of joint pain, stiffness and physical disability. It is widely accepted that arthritis patients tend to be weather sensitive. Historically, the pain-weather relationship has attracted interest since the classical Roman age..$^{10}$ In the past two decades, more and more research has been conducted, but results have turned out to be largely inconsistent. This area of uncertainty has contributed to the complexity of the original question: is there a relationship between weather and arthritis symptoms? If yes, what kind of relationship is it?

Conflicting findings regarding this issue arise at three different levels. First, as regard the question is weather associated with arthritis symptoms, some studies claimed that weather conditions, or at least some of the meteorological factors, did associated with patients' arthritis symptoms. ${ }^{1,10-15}$ However, others argued that their overall findings did not support this belief, or there might be association but it is not clinically significant. ${ }^{16}$ Some even concluded that weather sensitivity is a highly individual phenomenon, which means the association exists in some patients but cannot be found in others. ${ }^{17}$

Secondly, among the studies which support the idea that weather would influence arthritis symptoms, there are disagreements on which weather variables are involved. For studies focused on $\mathrm{OA}$ patients, some found that temperature is associated with pain, ${ }^{11,15}$ while some denied the association. ${ }^{10,12,13,18}$ The pain-humidity correlation was supported in one research, ${ }^{12}$ but failed to be supported in other two researches. ${ }^{10,11}$ The relationship between pain and precipitation was also controversial, which was proved by one study, ${ }^{15}$ but disproved by other four studies. ${ }^{11-13,18}$ Similarly, there is no consensus in RA researches. The association between temperature and pain in RA patients was found in some researches, ${ }^{10,14-16}$ but not in others. ${ }^{17}$ Also, some showed that pain and humidity was related, ${ }^{16}$ but others did not. ${ }^{10,14,17}$ Barometric pressure was considered to have influence on pain in some studies, ${ }^{15,16}$ but this belief was not supported by other studies. ${ }^{10,14,17}$ The findings of pain-sunshine association in RA patients conflicted as well, with one supporting it, $^{16}$ while another not supporting it. ${ }^{14}$ Thirdly,
Correspondence: Wai Chin Li, Department of Science and Environmental Studies, The Hong Kong Institute of Education, 10 Lo Ping Road, Tai Po, New Territories, Hong Kong.

Tel.: +852.2948.8630 - Fax: +852.2948 .7676 .

E-mail: waichin@ied.edu.hk

Key words: osteoarthritis; rheumatoid arthritis; barometric pressure; meteorology.

Acknowledgements: the authors would also like to thank Mr. Chan Yung Hau for the manuscript preparation. Financial support from the Department of Science and Environmental Studies of the Hong Kong Institute of Education is gratefully acknowledged.

Contributions: the authors contributed equally.

Conflict of interests: the authors declare no potential conflict of interests.

Received for publication: 21 March 2014.

Revision received: 28 April 2014.

Accepted for publication: 10 May 2014.

This work is licensed under a Creative Commons Attribution NonCommercial 3.0 License (CC BYNC 3.0).

(C) Copyright W.C. Li and Y. Zhang, 2014

Licensee PAGEPress, Italy

Rheumatology Reports 2014; 6:5398

doi:10.4081/rr.2014.5398

even among those who believed there was a correlation between two certain variables, the form of the correlation can be contradictory. For example, three studies proved a significant association between barometric pressure and $\mathrm{OA}$ pain, but one claimed the correlation was negative,,$^{10}$ and two found it was positive. ${ }^{11,15}$ Although a number of studies have been done in the latest two decades, questions relating to weather-symptom relationship in arthritis patients still remain nonconclusive.

\section{Objectives and importance}

Since the outcome of previous studies varied greatly and some findings were even contradictory, it would be necessary to review these studies and try to explain the causes of their differences. By doing so, not only some controversies can be clarified, but also shortcomings in research design can be avoided in future studies. The objectives of this paper are as follows: i) to review and summarize previous studies on weathersymptom relationship; ii) to compare and explain their differences; iii) to provide suggestions for future studies. 


\section{Results and Discussion}

A total of 12 reports discussing the relationship between weather and arthritis symptoms are included in this review, with 4 targeted at OA patients, 3 targeted at RA patients and 5 having a mixed sample of $\mathrm{OA}$ and RA patients.

Table 1 summarizes the major findings of each study, showing which weather factors were involved, which of them were proved to be symptom-related and which were not (Table 1). ${ }^{11-22}$ In Table 1, temperature and barometric pressure were taken into account in all 12 studies, and both of them were considered to

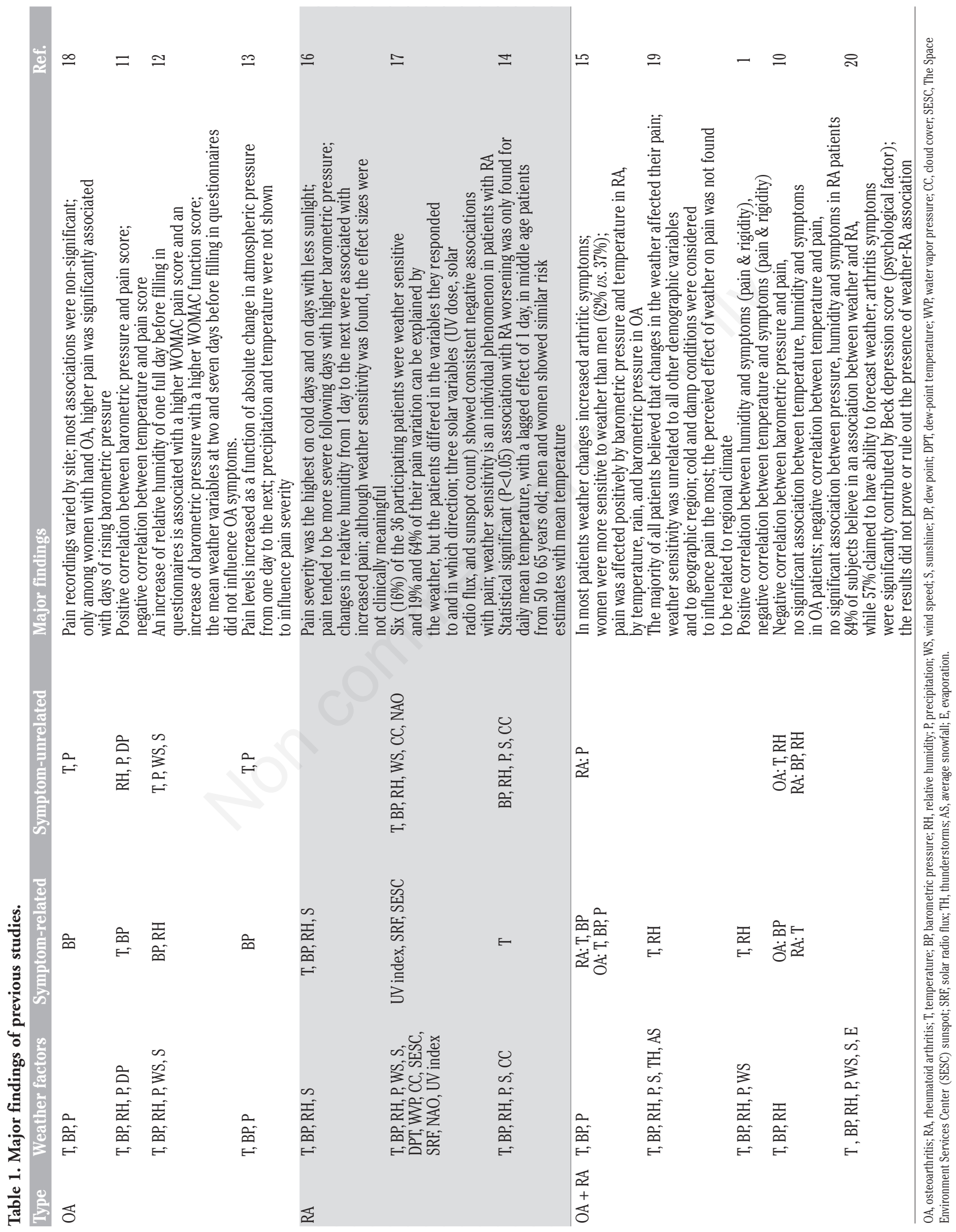


be symptom-related in 7 studies. However, barometric pressure and temperature were found unrelated to arthritis symptoms in 2 and 5 studies, respectively. Precipitation was mentioned in 10 studies, but half of them did not support the belief of rain-symptom association. As for relative humidity, although it was included in 9 of the 12 studies, its association with arthritis symptoms was confirmed by 4 studies and denied by 4 studies. Sunshine and wind speed were also considered, but few findings supported their association with arthritis symptoms ( $1 / 6$ and $0 / 4$ respectively). It seems that symptoms of arthritis patients are most likely to be influenced by pressure and temperature, while the impact of precipitation may be minimal (Table 2).

However, results would be more complicated if the data was stratified by patients' arthritis type (OA or RA). There are originally 4 studies focusing on $0 A$ patients and 3 on RA patients, but since two of the studies with mixed sample treated $\mathrm{OA}$ and RA separately, ${ }^{10,15}$ each of them can be regarded as two studies and added to the $\mathrm{OA}$ and RA catalogs. Previous findings about weather-symptom relationship stratified by arthritis type are as follows (Table 3 ).

It is clear from Table 3 that all of the 6 studies focusing on $\mathrm{OA}$ supported the belief that barometric pressure was associated with $\mathrm{OA}$ pain. 4 out of 6 studies showed that temperature and precipitation were not associated with $\mathrm{OA}$ pain. By contrast, temperature was considered to be pain-related among RA patients, with 4 out of 5 supporting this view. Relative humidity and barometric pressure seems to be pain-unrelated because their association with RA pain was denied by 3 out of 5 RA studies. Overall, based on the findings of 12 studies, OA pain is likely to be associated with barometric pressure while RA pain is likely to be associated with temperature. Temperature and precipitation do not seem to be related to $\mathrm{OA}$ pain, and humidity and barometric pressure do not seem to be related to RA pain. Although similarities can be found in these findings, most data still remain inconsistent and requires further investigation.

\section{Why differences occur? Factors influencing the relationship between weather and pain}

Weather-symptom association among arthritis patients has been extensively explored by researchers but most of the findings are controversial. The causes of those differences are not clear, but we try to explain why these differences could possibly occur from three perspectives: i) variable, ii) sample, and iii) methodology.

\section{Variable}

What kind of results one can get largely depends on what weather variables were set and how they were controlled. Weather variables varied from study to study. Among the 12 studies being reviewed, a total number of 17 weather variables were mentioned, but only barometric pressure and temperature were included in every study. Most studies used individual weather factors and investigated their effects on arthritis symptoms separately, but some suggested that weather variables are highly correlated so the combined effects need to be considered as well. Actually, in a research about weather and pain in fibromyalgia (not included in the 12 studies), researchers used a composite daily weather variable which was calculated from indices like barometric pressure, temperature, humidity, sunlight, cloudiness etc. ${ }^{21}$ Moreover, an earlier study which was conducted in a climate-controlled chamber showed that arthritis pain increased only when humidity increased and pressure decreased at the same time. There was no change in patients' pain perception if only one of them changed. ${ }^{22}$

In addition, the same weather variable may have subtle differences in different studies, which may also lead to different results. For example, the effects of sunlight on arthritis symptoms were considered in 6 of the 12 studies, but some measured sun hours of a day while some calculated average days of sun- shine during a period. Also, with the same attempt to investigate the association between weather and symptoms, some studies took into account the direction of change in weather indices while some did not, and that may explain inconsistent results, too. For example, conflicting outcomes were found in the relationship between barometric pressure and $\mathrm{OA}$ pain. Some claimed a positive correlation between them, ${ }^{11,15,18}$ while some thought it should be a negative correlation. ${ }^{10}$ However, one study indicated that it was the absolute change in barometric pressure that caused increased $0 \mathrm{~A}$ pain. ${ }^{13}$ That means, regardless of the direction of pressure change, $\mathrm{OA}$ pain would increase as a result of pressure change. If that argument could be supported by further studies, inconsistency in results about painpressure association would become understandable.

\section{Patients}

Differences in the sample of patients would also cause different results. First of all, it is clear from Table 3 that weather variables had different influence on different types of arthritis (OA and RA). Barometric pressure was likely to be associated with $0 A$ pain rather than RA pain, and temperature was likely to be associated with RA pain instead of OA pain. Therefore, results of studies with mixed sample may be confounded by such difference.

Secondly, gender and pain sites may also

Table 2. Meteorological variables and their correlation with arthritis symptoms.

\begin{tabular}{lccc} 
Variable & Mentioned (times) & Symptom-related & Symptom-unrelated \\
Barometric pressure & 12 & 7 & 2 \\
Temperature & 12 & 7 & 5 \\
\hline Precipitation & 10 & 1 & 5 \\
Relative humidity & 9 & 4 & 4 \\
\hline Sunshine & 6 & 1 & 2 \\
Wind speed & 4 & 0 & 2 \\
\hline
\end{tabular}

Table 3. Meteorological variable-symptom correlation stratified by arthritis types.

\begin{tabular}{lll} 
& OA $(6)$ & RA (5) \\
Symptom-related & & \\
Barometric pressure & 6 & 4 \\
Temperature & 2 & 1 \\
Relative humidity & 1 & 0 \\
Precipitation & 1 & 0 \\
Wind speed & 0 & 1 \\
Sunshine & 0 & \\
Symptom-unrelated & & 3 \\
Barometric pressure & 0 & 1 \\
Temperature & 4 & 3 \\
Relative humidity & 2 & 2 \\
Precipitation & 4 & 1 \\
Wind speed & 1 & 1 \\
Sunshine & 2 & \\
\hline
\end{tabular}

OA, osteoarthritis; RA, rheumatoid arthritis. 
influence the weather-symptom association. When describing the sample, 8 of the 12 studies mentioned gender of their participants, ${ }^{1,11,14-19}$ and 8 of them reported the exact gender ratio.,11,14,16,17 This may due to statistical recording, because most of the studies did not consider the role of gender in their analysis. However, some studies still indicated that weather sensitivity varied between the two genders, and women were more sensitive to weather than men. ${ }^{15}$ The site of pain was specified in 4 of the 12 studies, ${ }^{11-13,18}$ with two having hip OA participants only, ${ }_{12,13}$ one having knee OA participants only and one having participants with OA on neck, hand, shoulder, knee and foot. ${ }^{11,18}$ The study with patients having specified but different pain sites claimed that pain scores varied by site, and most of the weather-symptom associations were non-significant except among women with hand 0A. ${ }^{18}$ If such association only existed in certain sites of arthritis, findings of different studies would have conflicts.

Another important factor in sampling is participants' geographic location of residence. Obviously, the weather of a region is related to the local climate. From a long-term perspective, weather conditions in a wet, cold climate can be very different from that in a dry and warm climate. If weather was associated with arthritis symptoms, would the association be influenced by regional climate? Is symptoms affected by weather in the same way, to the same extent, in different parts of the world? Those questions still remain unclear. A research showed that from a questionnaire survey done in 4 cities in U.S. the influence of weather on arthritis was not found to be associated with regional climate, but that has not been proved by scientific evidence.

There are other sample-related factors that may influence the research findings of weather-symptom relationship, such as participants' age, severity of disease and meditation usage, which can be highly individual. Since little is known about the effect of demographic variables on arthritis symptoms, it is difficult to tell whether the inconsistent findings of previous studies are caused by differences in sample.

\section{Methodology}

There is a wide variety of methodological differences in the previous studies but this article mainly reviewed the differences in data collection and data analysis. Two kinds of data were collected in each study: weather indices and symptom (pain, stiffness, function) scores. Weather indices were mostly recorded from the national or local weather stations, but regarding symptom scores, some used standardized format such as Visual Analogue Scale (VAS), ${ }^{10,12,13,16-18,20}$ or The Western Ontario and McMaster Universities Arthritis Index (WOMAC), ${ }^{11,12}$ while some designed their own questionnaires and measurements. ${ }^{1,15,19,20}$ As for the frequency of data recording, some do it once a day, ${ }^{10-12,14-17,20}$ some on weekly basis, ${ }^{18}$ some do it every three months, ${ }^{12}$ and some recorded 4 times per day and calculated the mean value. ${ }^{1}$ The total duration of observation also ranged from dozens of days to several years. For data analysis, the methods adopted by previous studies varied as well. One of the studies compared the major analytic methods in other studies, which include simple correlation analysis, aggregated analysis and time series analysis. According to that article, simple correlation analysis is likely to overestimate the influence of weather while aggregated analysis tends to underestimate that influence. Since the association between weather and arthritis symptoms is complex and hard to identify, time series methodology is recommended by the authors. ${ }^{17}$ Whether time series is the best way to analyze weather-symptom association is still debatable, but at least it can be seen that different ways of data analysis can possibly lead to different results.

\section{Limitations}

Researches about weather-symptom relationship being mentioned in this article have similar limitations. Firstly, most of the studies had small sample size which was under $100.1,10,13,15-17,20$ In two of the studies, the number of participant was less than $40,1,17$ which was not very representative and reliable. Secondly, samples were heterogeneous in term of arthritis type, gender, pain sites, severity of disease, meditation usage etc., and that heterogeneousness may act as confounders of weather effects. Moreover, the observation periods in the studies were not very long, most of which only lasted for 1 to 2 months. If weather effects on arthritis symptoms were influenced by seasonal changes, which were indeed proved by some studies, findings based on short-term observations would be unable to present the association in a comprehensive way. Another problem is about how to control weather variables. In reality, weather variables like temperature, humidity and barometric pressure are highly interrelated and can hardly be controlled and studied separately. Higher level of pain and physical incapacity may be caused by some combined effects such as a combination of temperature decrease and humidity increase. Therefore, it would be difficult to argue that certain weather variables affect arthritis symptoms independently. On the other hand, if we group up some variables to become a new factor, e.g. temperature + humidity, we would probably have difficulty assigning meaning to it.

Most importantly, psychological misattribu- tion was seldom assessed in the previous studies. From the daily experience, arthritis patients have the anecdotal belief that weather has influence on their symptoms, and the most prevalent belief is that joint pain gets worse when it is cold and damp. Because of that, even if the participants were not aware of the hypothesis of the study, they might still associate their arthritis symptoms with observable weather factors and reflect that suspicion on the questionnaires or dairies that they were asked to finish. That would obviously bias some associations explored by researchers.

\section{Further investigation and recommendation}

So far, there still is lack of large-scale, largesample, long-term, well-controlled and wellconducted researches to prove the weathersymptom relationship and psychological factors such as patients' anecdotal beliefs have not been fully considered in the explanation. Future studies are supposed to improve their research design and conduct in those aspects, but at the same time, efforts should be made to increase the comparability of different studies and try to understand the underlying mechanism of weather-symptom association.

Previous studies varied greatly in terms of variable, sample and methodology, and that would probably lead to inconsistent results. The lack of uniform method has increased the difficulty of comparison. Therefore, it is suggested that researchers should come up with some standardized methods to enhance the comparability of different studies, for example, whether do the recording in the morning or before bed, whether use VAS or WOMAC, and whether adopt simple correlation analysis or time series analysis etc. By doing so, the generalizability of one study could be improved as well. Arthritis is a common disease but its pathology remains largely unknown. Hopefully, there might be well-designed, well-conducted and comparable studies in the future, but without knowing the mechanism behind the weather-symptom relationship these studies are not going to be of clinical significance. Some studies tried to explain the biological mechanism of weather-symptom association. For example, intra-articular pressure is normally lower than barometric pressure, but one study found that when barometric pressure decreases and equals the intraarticular pressure, the hip joint shows $8 \mathrm{~mm}$ of subluxation. ${ }^{23}$ That may explain why barometric pressure has influence on arthritis symptoms. Further studies are supposed to explore not only the subjective weather-symptom association, but also find out the objective mechanisms in action. 
To sum up, whether arthritis symptoms are influenced by weather is still non-conclusive, but relevant researches have been conducted to investigate the relationship between weather conditions and arthritic symptoms. Most of the results have showed a possible relationship between them but the significance of the relationship is still controversial and the correlations between each two variables remain debatable. Essentially, answering the question can arthritis predict weather requires deeper exploration of the relationship between meteorological factors and arthritic symptoms, and such exploration can be valuable. If the relationship can be clearly defined and proved in the future, as researchers mentioned, it will contribute to the non-pharmacological treatments of arthritic patient and help them live a better life by avoiding harmful weather factors. ${ }^{10}$ Therefore, researches with large scale, large sample, long observation period and well-controlled variables are required in the future, in order to provide supplementary treatments and improve arthritic patients' quality of life.

\section{References}

1. Aikman H. The association between arthritis and the weather. Int $\mathrm{J}$ Biometeorol 1997;40:192-9.

2. Loeser RF, Goldring SR, Scanzello CR, Goldring RB. Osteoarthritis: a disease of the joint as an organ. Arthritis Rheum 2012;64:1697-707.

3. Goldring RB, Goldring SR. Osteoarthritis. J Cell Physiol 2007;213:626-34. Orthopaedics 2012;36:643-6.
4. Schett G, Firestein GS. Mr outside and Mr inside: classic and alternative views on the pathogenesis of rheumatoid arthritis. Ann Rheum Dis 2010;69:787-9.

rheumatoid arthritis patients: the possible effect of temperature. Reumatol Clín 2003;9:226-8.

5. Bugatti S, Manzo A, Caporali R, Montecucco C. Inflammatory lesions in the bone marrow of rheumatoid arthritis patients: a morphological perspective. Arthritis Res Ther 2012;14:229.

6. Hitchon CA, El-Gabalawy HS. The synovium in rheumatoid arthritis. Open Rheumatol J 2011;5:107-14.

7. Bugatti S, Manzo A, Bombardieri M, et al. Synovial tissue heterogeneity and peripheral blood biomarkers. Curr Rheumatol Rep 2011;13:440-8.

8. McInnes IB, Schett G. The pathogenesis of rheumatoid arthritis. New Engl J Med 2011;365:2205-19.

9. Arthritis Foundation. The heavy burden of arthritis in the U.S., 2011. Available from: http://www.arthritis.org/files/images/news room/Arthritis_Prevalence_Fact_Sheet_531-11.pdf

10. Vergés $\mathrm{J}$, Montell $\mathrm{E}$, Tomàs $\mathrm{E}$, et al. Weather conditions can influence rheumatic diseases. Paper presented at the Proc West Pharmacol Soc 2004;47:134-6.

11. McAlindon T, Formica M, Schmid CH, Fletcher J. Changes in barometric pressure and ambient temperature influence osteoarthritis pain. Am J Med 2007;120: 429-34.

12. Dorleijn P, Bouterse M, Luijsterburg P, et al. 333 effect of weather conditions on patients with hip osteoarthritis. Osteoarthritis Cartilage 2011;19:S152.

13. Brennan SA, Harney T, Queally JM, et al. Influence of weather variables on pain severity in end-stage osteoarthritis. Int

14. Abasolo L, Tobías A, Leon L, et al. Weather conditions may worsen symptoms in
15. Guedj D, Weinberger A. Effect of weather conditions on rheumatic patients. Ann Rheum Dis 1990;49:158-9.

16. Gorin AA, Smyth JM, Weisberg JN, et al. Rheumatoid arthritis patients show weather sensitivity in daily life, but the relationship is not clinically significant. Pain 1999;81:173-7.

17. Smedslund G, Mowinckel P, Heiberg T, et al. Does the weather really matter? A cohort study of influences of weather and solar conditions on daily variations of joint pain in patients with rheumatoid arthritis. Arthritis Care Res 2009;61:1243-7.

18. Wilder F, Hall B, Barrett J. Osteoarthritis pain and weather. Rheumatology 2003;42: 955-8.

19. Jamison RN, Anderson KO, Slater MA. Weather changes and pain: perceived influence of local climate on pain complaint in chronic pain patients. Pain 1995; 61:309-15.

20. Çay HF, Sezer I, Firat MZ, Kaçar C. Which is the dominant factor for perception of rheumatic pain: meteorology or psychology? Rheumatol Int 2011;31:377-85.

21. Fors E, Sexton H. Weather and the pain in fibromyalgia: are they related? Ann Rheum Dis 2002;61:247-50.

22. Hollander J. Division of oceanography and meteorology: the controlled-climate chamber for study of the effects of meteorological changes on human diseases. Trans N Y Acad Sci 1961;24:167-72.

23. Wingstrand H, Wingstrand A, Krantz P. Intracapsular and atmospheric pressure in the dynamics and stability of the hip: a biomechanical study. Acta Orthopaedica 1990;61:231-5 